\title{
Editorial
}

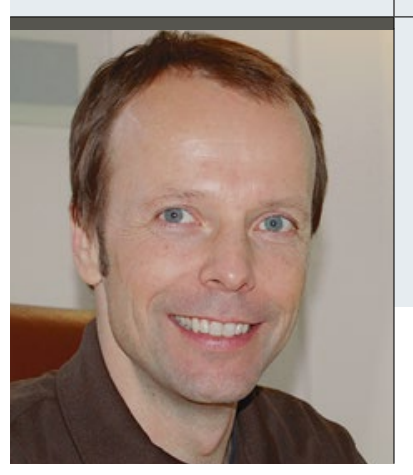

"Die Forschungsergebnisse der letzten Jahre zur

Bewegungstherapie sind eindeutig."

\author{
Dr. med. Thomas Lukowski
}

Facharzt für Psychiatrie und Psychotherapie, München

\section{Bewegung auf Rezept}

W er rastet, der rostet nicht nur, sondern vergibt immense Möglichkeiten zur Prophylaxe und Behandlung psychischer Erkrankungen. Die Forschungsergebnisse der letzten Jahre, in Zusammenschau mit der praktischen Erfahrung im ambulanten wie stationären Bereich bezüglich der Wirksamkeit einer fundierten Bewegungstherapie, sind eindeutig. So kann die Bewegungstherapie - neben der Psycho- und der Psychopharmakotherapie - als dritte Säule der Behandlung psychischer Erkrankungen genannt werden.

Leider waren uns die Kollegen der somatischen Medizin bis dato einen Schritt voraus. Schon in den 1990er-Jahren wurden von Hollmann und Strüder (Deutsche Sporthochschule Köln) bahnbrechende Arbeiten zum Einfluss von Bewegung auf physiosowie psychologische Effekte im Gehirn unternommen und es gibt „Sport auf Rezept“ (KBV 2014), jedoch nur bei körperlichen Erkrankungen oder für behinderte Menschen. Aber wir holen gewaltig auf. Ich erinnere hier nur an aussagekräftige Untersuchungen zur Prophylaxe kognitiver Störungen [Sattler et al. 2011], von Depression und Suizidalität bei Jugendlichen [Babiss/Gangwisch 2009], zur Verbesserung kognitiver Leistungen bei schizophrenen Patienten [Oertel-Knöchel et al. 2014] und der vielschichtigen antidepressiven Wirksamkeit von Bewegungstherapie [Barbour et al. 2007].

Nun widme ich mich seit vielen Jahren dem therapeutischen Klettern und es liegen immer mehr quantitative sowie qualitative Erhebungen zur Wirksamkeit vor [z. B. Paulik 2016]. Ganz aktuell seien hier die Projekte der Kolleginnen Jana Strahler vom Fachbereich Psychologie und Sportwissenschaft der Justus-Liebig-Universität Giessen und Maria Kleinstäuber, Philipps-Universität Marburg, sowie Katharina Luttenberger, Psychiatrische und Psychotherapeutische Klinik am Universitätsklinikum Erlangen, genannt. Die Grundlagenforscherinnen Strahler und Kleinstäuber beschäftigen sich mit psychometrisch wie physiologisch fassbaren Auswirkungen des Boulderns (Klettern in Ab- sprunghöhe) auf die emotionale Gestimmtheit ihrer Probanden. Direkte Messungen von Speichelkortisol und -amylase (als Marker der Stressachse) werden angestrebt. Luttenberger widmet sich in ihrer bereits zweiten Therapiestudie KuS (Klettern und Stimmung) dem direkten Vergleich einer Bouldertherapie (standardisiertes Manual) versus ambulante Gruppen- und Bewegungstherapie (www. studiekus.de). Ich bin schon sehr auf die Ergebnisse gespannt. Auch in der Öffentlichkeit wächst das Interesse an dieser innovativen Therapieform (Bayerisches Fernsehen/Mediathek: Sendung vom 11. Juli 2017, „Gesundheit“, Therapeutisches Klettern im Hochseilgarten). Diejenigen, die sich genauer mit dem Thema beschäftigen möchten, können sich jetzt schon auf mein Buch „Klettern in der Therapie“ (Ernst Reinhardt Verlag, München, Herbst 2017) freuen.

Und auch die Kollegen des Referates „Sportpsychiatrie und -psychotherapie" der DGPPN sind aktiv geworden. Andreas Ströhle, Charité Berlin, und Kollegen haben gerade ein Forschungsvorhaben zu „Bewegung auf Rezept“ für psychisch kranke Menschen aus der Taufe gehoben. Alle Genannten freuen sich auf einen regen Austausch und/oder Beteiligung an ihren Projekten.

Ihnen, geschätzte Kolleginnen und Kollegen, lege ich aber genauso gerne die „klassischen“ Themen unseres Fachbereichs ans Herz, die wie immer profund und äußerst lesbar in dieser Ausgabe von DNP behandelt werden. Auszugsweise seien hier die Fortbildungsbeiträge genannt: Gerontopsychiatrische Aspekte in der Versorgung, Neuropalliative Care Palliativmedizin bei Patienten mit Parkinson-Erkrankungen, Lithium in der Langzeittherapie der bipolaren Störung - was gibt es Neues?

So wünsche ich ein genüssliches und erkenntnisreiches Lesen, ein frohes Schaffen und Bewegen, denn: Sich regen bringt Segen.

Ihr

\section{Thomas Lukowski}

